\title{
Actividad física y aptitud física en niños del sector urbano y rural de Lebrija, Santander
}

\author{
Physical activity and physical fitness in children in the urban and rural sector of \\ Lebrija, Santander
}

Diego Fernando Álvarez'1, Luis Gabriel Rangel-Caballero²

Citación: Álvarez DF, Rangel-Caballero LG. Actividad física y aptitud física en niños del sector urbano y rural de Lebrija, Santander. Ustasalud 2019;18: 28-38.

Licencia Creative Commons

\section{(c) (1) () $\Theta$} lo tanto, los lectores pueden acceder libremente a los artículos en su formato .pdf, igualmente podrán descargarlos y difundirlos; sin embargo no podrán modificarlos o alterarlos, adicionalmente se debe reconocer la autoría de las personas que figuran en las publicaciones, pero estas no podrán ser comercializadas.

1 Profesional en Cultura Física, Deporte y Recreación, docente Facultad de Cultura Física, Deporte y Recreación, Universidad Santo Tomás, Bucaramanga, Colombia

2 Magíster en Actividad Física: Entrenamiento y Gestión Deportiva, docente Facultad de Cultura Física, Deporte y Recreación, Universidad Santo Tomás, Bucaramanga, Colombia

Autor de correspondencia:

Luis Gabriel Rangel-Caballero

Correo electrónico:

dcultu@ustabuca.edu.co

\section{Resumen}

Objetivo: Determinar las diferencias entre el sector urbano y rural en los niveles de actividad física y aptitud física de niños escolarizados de un colegio de Lebrija, Santander. Materiales y métodos: Se realizó un estudio observacional analítico de corte transversal en 115 estudiantes (60 niños, 55 niñas) de un colegio de Lebrija, Santander. Se solicitó consentimiento informado a los padres de los participantes del estudio. Para valorar la actividad física se utilizó la Encuesta Mundial de Salud a Escolares, en el caso de la aptitud física fueron utilizadas las pruebas de la batería Alpha-Fitness. Las variables fueron analizadas en medidas de tendencia central o frecuencias según su naturaleza. Se utilizó la prueba t de Student, U de Mann Whitney y exacto de Fischer para establecer diferencias estadísticamente significativas. Resultados: El promedio de edad de los participantes fue de 9,78 años (DE 1,16). En cuanto al lugar de residencia, no se presentaron diferencias estadísticamente significativas en las variables relacionadas con la aptitud física, aunque los niños del sector rural reportaron mejores niveles de fuerza de prensión manual, fuerza explosiva del tren inferior y capacidad aeróbica. Los participantes del sector urbano reportaron ser más activos físicamente que los del sector rural $(p<0,001)$. Conclusiones: No se encontró una diferencia estadísticamente significativa según el lugar de residencia en relación con los componentes de la aptitud física. En caso contrario, los niños que viven en el sector urbano reportaron ser físicamente más activos que los niños del sector rural.

Palabras clave: Actividad motora, aptitud física, niño, población rural, población urbana.

\section{Abstract}

Objective: To establish differences between urban and rural sector in physical activity and physical fitness in children of a school from Lebrija, Santander. Methods: An analytical cross-sectional study was carried out with 115 students ( 60 boys, 55 girls) that attended a school in Lebrija, Santander. Written consent was solicited to parents of participants in the study. To assess physical activity was utilized Global School-Based Student Health Survey, in the case of physical fitness, Alpha-Fitness battery was applied. Variables were analyzed in frequencies or central tendencies measures according to its nature. Fischer exact, T- Student and U-Mann Whitney tests were utilized to establish a difference statistically significant. Results: Mean age of participants was of 9,78 years (ED: 1,16). Regarding to place of living, a difference statistically significant was not found in variables related to physical fitness, although boys from rural sector reported higher levels of handgrip strength, lower limb power and aerobic capacity. Participants of urban sector reported being more physically active than those of rural sector $(p<0,001)$. Conclusions: A Difference statistically significant was not found according to place of residence in relation to physical fitness components. On the other hand, children who live in urban sector reported being more physically active than those who live in the rural sector.

Key words: Motor activity, physical fitness, child, rural population, urban population.
Recibido para publicación: 19 de octubre de 2019 Aceptado para publicación: 26 de noviembre de 2019 


\section{INTRODUCCIÓN}

La Organización Mundial de la Salud (OMS) se refiere a la actividad física como cualquier movimiento del cuerpo ocasionado por el aparato locomotor, que da como resultado un gasto de energía corporal. La actividad física está estrechamente relacionada con la salud, por esta razón su práctica ha sido motivo de estudio de muchos investigadores ${ }^{1}$. La actividad física puede traer diferentes beneficios en el bienestar psicológico ${ }^{2}$, además de esto, su práctica regular y adecuada es un factor importante a la hora de ayudar a tratar problemas de sobrepeso, obesidad y a disminuir múltiples factores de riesgo de adquirir enfermedades crónicas ${ }^{3}$.

En el caso específico de los niños, la cantidad de tiempo que le dedican a la realización de actividad física está estrechamente relacionada con el tiempo que utilizan para jugar al aire libre ${ }_{4}$. Además de esto, la práctica regular de cualquier tipo de actividad física en niños escolares de básica primaria depende de múltiples factores, como por ejemplo la participación en clubes deportivos, la disponibilidad de implementos para la realización de actividad física y el tiempo que ocupan viendo televisión ${ }^{5}$.

La aptitud física hace referencia a la capacidad que tiene el organismo de realizar actividad física por medio de las diferentes funciones fisiológicas del cuerpo humano; al ser este un pronóstico de la expectativa de vida, la calidad de vida de las personas y el riesgo de padecer enfermedades cardiovasculares. Esto se debe a que unos óptimos niveles de aptitud física aportan al mejoramiento de la salud física y mental de las personas ${ }^{6}$.

La aptitud física se relaciona con los hábitos de vida y los niveles de actividad física de una población específica. Por esta razón, con su valoración se puede obtener información sobre el estado de salud y la calidad de vida de determinada población. Esta información es considerablemente importante a la hora de proponer, promover y orientar diferentes programas de actividad física, ya sean enfocados al desarrollo de la salud o de la aptitud deportiva ${ }^{7}$.

Se han realizado estudios en países como Estados Unidos $^{8}$, India ${ }^{9}$ y México ${ }^{10}$, donde se compararon los niveles de actividad física y aptitud física en niños habitantes de zonas urbanas y rurales, sin poder establecer un lugar de residencia que permita tener mayores niveles de actividad física y aptitud física a nivel global.

La infancia y la adolescencia son periodos muy importantes en la adquisición de los estilos de vida que se van a llevar más adelante en la adultez. En este ciclo de la vida, los niños y adolescentes tienen que lidiar con comportamientos y experiencias totalmente distintas a las que acostumbraban a tener antes. Esta situación se da a raíz del aumento de los vínculos sociales y las relaciones interpersonales, las cuales son esenciales en la formación de hábitos y conductas saludables a futuro ${ }^{11}$.

La práctica habitual de actividad física en los niños y adolescentes está ajustada a la disponibilidad que tengan para acceder a instalaciones adecuadas para el correcto aprovechamiento del tiempo libre, además está estrechamente relacionada con el clima que posea el lugar, las distancias que haya que recorrer, los medios de transporte que haya que usar, el diseño de las viviendas donde habitan y la organización a su alrededor. Junto con esto, la cantidad de niños que se desplazan hacia el colegio caminando o utilizando la bicicleta como medio de transporte, va a depender de factores como la ubicación de las instituciones educativas y la seguridad del camino, influyendo en la realización de actividad física como medio para transportarse hacia el colegio ${ }^{12}$.

La actividad física y la aptitud física en entornos rurales y urbanos han sido estudiadas en muchos países, encontrando una gran variabilidad en los resultados de cada investigación. Esto sucede porque existen diferencias en los aspectos políticos, económicos y del entorno entre un lugar y otro. Además de esto, hay que tener en cuenta que existen muchos factores que pueden determinar la práctica de actividad física dependiendo del lugar en el que habiten las personas.

El objetivo de este trabajo fue establecer si existen o no diferencias en los niveles de actividad física y aptitud física en niños escolarizados del sector rural y urbano, de 8 a 13 años del Colegio Integrado Nuestra Señora de las Mercedes. 


\section{MATERIALES Y MÉTODOS}

Se realizó un estudio analítico de corte transversal. Fue llevado a cabo un muestreo por conveniencia y la muestra estuvo conformada por 115 estudiantes escolarizados del colegio en mención, matriculados para el año 2017 en los cursos de tercero a quinto grado de básica primaria, y que habitaban en zonas urbanas y rurales del municipio de Lebrija, Santander (Colombia). Los participantes no debían tener algún riesgo ósteo-muscular o cardiovascular, además debían acceder de forma voluntaria (por medio del asentimiento informado) y debían ser autorizados por sus padres (por medio del consentimiento informado) para participar del estudio.

Inicialmente, se elevó una solicitud por escrito al rector del colegio para contar con la aprobación de la institución educativa. Después se realizó una reunión informativa con los estudiantes participantes y padres de familia para describir los objetivos del estudio y realizar la entrega del consentimiento y asentimiento informado.

El día de las valoraciones se confirmó que los participantes hubiesen entregado los documentos anteriormente descritos y finalmente, los que cumplieron los criterios de elegibilidad participaron voluntariamente de las pruebas. Para establecer el lugar de residencia, se solicitó esta información por escrito a los padres de familia en el documento de consentimiento informado y los investigadores teniendo en cuenta el Plan de Ordenamiento Territorial del Municipio clasificaron a los participantes en sector urbano o sector rural.

Primero, se aplicó la Encuesta Mundial de Salud a Escolares (EMSE) para evaluar los niveles de actividad física ${ }^{13}$; este instrumento fue aplicado utilizando el método de entrevista directa. Posteriormente, se realizó un calentamiento general de quince minutos con ejercicios de movilidad articular, activación cardiovascular y estiramiento. Luego del calentamiento, se procedió a la aplicación de la batería ALPHA-Fitness al tener en cuenta el modelo de seguridad y la secuencia recomendada para su administración, se describe a continuación ${ }^{14}$ :
Peso y altura (IMC). Para determinar el peso se utilizó una báscula TANITA UM-061 y para el caso de la talla, un tallímetro SECA 213.

Fuerza de prensión manual. Se valoró con el uso de un dinamómetro de agarre ajustable Takei 5001 (TKK 5001 Grip D; Takei, Tokio Japan) y con una regla tabla para calcular el agarre preciso se valoró la fuerza isométrica de los miembros superiores. El participante, con el agarre calculado previamente con la regla-tabla, apretó de forma continua por tres segundos con ambas manos de manera alterna en dos ocasiones con un corto descanso entre cada valoración. Se tomó la media en kilogramos de los mejores registros de cada mano y se emplearon los valores de referencia de la batería para determinar su nivel ${ }^{15}$.

Salto de longitud sin impulso. El estudiante se puso en bipedestación con los pies separados al ancho de los hombros detrás de la línea de inicio. Desde esa posición el estudiante saltó de manera horizontal, hacia delante lo más lejos posible. Se registró la máxima distancia lograda, teniendo como punto de referencia al terminar el salto la parte posterior del talón más atrasado. Se hicieron dos intentos y se reportó la medida en centímetros del mejor de los dos intentos. De esta manera se valoró la fuerza explosiva de los miembros inferiores. Se emplearon los valores de referencia de la batería para determinar su nivel ${ }^{16}$.

Velocidad agilidad $4 \times 10 \mathrm{~m}$. Mediante esta prueba se evaluó de forma integral la velocidad de movimiento, la agilidad y la coordinación. El participante se ubicó detrás de la línea de salida, a la señal, el estudiante corrió a la máxima velocidad posible hasta completar el recorrido de 40 metros hasta el lugar señalado como llegada. La prueba se realizó en dos ocasiones y fue reportado en segundos el mejor intento. Para establecer el nivel se tuvieron en cuenta los valores de referencia de la batería ${ }^{16}$.

Test de ida y vuelta de 20 metros. Con esta prueba se estableció la capacidad aeróbica mediante el consumo máximo de oxígeno $\left(\mathrm{VO}_{2}\right.$ máx.). Cada estudiante cubrió una distancia de 20 metros en doble sentido, ida y vuelta, tocando con el pie la línea de base que se encuentra localizada al final de cada uno de los extremos al mismo tiempo de la señal sonora emitida por una grabación. La frecuencia de la señal sonora incrementó en $0,5 \mathrm{~km} / \mathrm{h}^{-1}$ cada minuto comenzando con una 
velocidad de $8,5 \mathrm{Km} / \mathrm{h}^{-1}$. La prueba finalizó cuando el participante no pudo tocar por dos veces consecutivas la línea antes de la señal sonora o cuando decidió abandonar por fatiga. Se reportó el último medio estadio o parlier completado y con este registro se determinó el consumo máximo de oxígeno $\left(\mathrm{VO}_{2}\right.$ máx) expresado en $\mathrm{ml} / \mathrm{kg}^{-1} / \mathrm{min}^{-1}$ obtenido a través de la fórmula de Leger $^{17}$. Para establecer los niveles de este componente se tuvieron en cuenta los criterios de la batería ${ }^{16}$.

Adicionalmente, se analizaron otras variables como el sexo, la edad, el grado de escolaridad. Un profesional y un estudiante de Cultura Física, Deporte y Recreación fueron los encargados de la recolección de la información y aplicación de las pruebas, los cuales fueron debidamente capacitados y entrenados. Las valoraciones se realizaron en las instalaciones del colegio, en el tiempo de clase de educación física y en presencia del docente de la institución educativa y personal de enfermería.

\section{Análisis estadístico}

Se analizaron las variables de interés en la población a estudiar. Las variables categóricas se describieron con frecuencias y porcentajes. La evaluación de la distribución de las variables continuas se hizo por medio de la prueba de Shapiro Wilk. Las variables de interés se expresaron como promedios y desviación estándar cuando se presentó una distribución normal, en caso contrario, se describieron como mediana y rango intercuartílico. Con el fin de determinar la existencia de diferencias estadísticamente significativas según el sexo y el lugar de residencia, se usaron las pruebas $t$ de Student y U de Mann-Whitney para variables continuas y, para el caso de las variables categóricas se utilizó la prueba exacta de Fisher. El nivel de significancia fue de 0,05.

\section{Consideraciones éticas}

Según la Resolución 08430 de 1993 del Ministerio de Salud de la República de Colombia, esta investigación se clasifica como "Riesgo Mínimo", por lo tanto los padres de familia autorizaron la participación de los niños a través de la firma del consentimiento informado, el cual presentaba de manera clara la información en relación con la investigación descrita en el artículo 15 de la resolución en mención.

\section{RESULTADOS}

El promedio de edad de los participantes fue de 9,78 años, existiendo una mayor participación de los niños $(52,17 \%)$ sobre las niñas $(47,83 \%)$. En cuanto al grado de escolaridad, el $36,52 \%$ eran estudiantes de cuarto primaria, el $32,17 \%$ del grado tercero y el $31,30 \%$ del grado quinto. En relación con el lugar de residencia, el 63,48\% de los escolares evaluados del Colegio Integrado Nuestra Señora de las Mercedes, viven en la zona urbana del municipio de Lebrija (Tablas 1 y 2).

Tabla 1. Descripción de las características sociodemográficas de actividad física y aptitud física de los participantes $(\mathrm{n}=115)$

\begin{tabular}{|c|c|}
\hline Características & n (\%) \\
\hline \multicolumn{2}{|l|}{ Sexo } \\
\hline Masculino & $60(52,17)$ \\
\hline Femenino & $55(47,83)$ \\
\hline \multicolumn{2}{|l|}{ Grado } \\
\hline Tercero primaria & $37(32,17)$ \\
\hline Cuarto primaria & $42(36,52)$ \\
\hline Quinto primaria & $36(31,30)$ \\
\hline \multicolumn{2}{|l|}{ Lugar de residencia } \\
\hline Urbano & $73(63,48)$ \\
\hline Rural & $42(36,52)$ \\
\hline \multicolumn{2}{|l|}{ Estado nutricional (IMC) } \\
\hline Zona saludable & $85(73,95)$ \\
\hline Necesita mejora/riesgo cardiovascular & $23(20,00)$ \\
\hline Bajo peso & $7(6,09)$ \\
\hline \multicolumn{2}{|l|}{$\begin{array}{l}\text { Cumplimiento de las recomendaciones de } \\
\text { la OMS sobre actividad física para la salud } \\
{ }_{(n=91)}\end{array}$} \\
\hline Activo & $39(42,86)$ \\
\hline Inactivo & $52(57,14)$ \\
\hline \multicolumn{2}{|l|}{$\begin{array}{l}\text { Realización de actividad física relacionada } \\
\text { con el transporte }(n=90)\end{array}$} \\
\hline No realiza & $30(33,33)$ \\
\hline Entre menos de 10 minutos y 30 minutos & $45(50,00)$ \\
\hline Más de 30 minutos & $15(16,66)$ \\
\hline \multicolumn{2}{|l|}{ Conducta sedentaria } \\
\hline Menos de una hora al día - 4 horas al día & $77(85,55)$ \\
\hline Cinco horas al día - 8 horas al día & $10(11,11)$ \\
\hline Más de 8 horas al día & $3(03,33)$ \\
\hline
\end{tabular}

$I M C$ : Índice de Masa Corporal; ${ }^{*}$ Determinado por la Encuesta Mundial de Salud a Escolares (EMSE). 
Tabla 2. Descripción de las características sociodemográficas de actividad física y aptitud física de los participantes $(\mathrm{n}=115)$

\begin{tabular}{lcc}
\hline \multicolumn{1}{c}{ Características } & $\begin{array}{c}\text { Promedio/ } \\
\text { Mediana } \dagger\end{array}$ & DE $\$ / \mathbf{R I}^{* *}$ \\
\hline Edad & 9,78 & 1,16 \\
Talla $(\mathrm{cm})$ & 138,00 & 7,47 \\
\hline Peso $(\mathrm{kg})$ & $32,9 \dagger$ & $9,30^{* *}$ \\
IMC $\left(\mathrm{Kg} / \mathrm{m}^{2}\right)$ & $16,90 \dagger$ & $3,20^{* *}$ \\
\hline Fuerza de prensión manual $(\mathrm{kg})$ & $16,00 \dagger$ & $5,00^{* *}$ \\
\hline $\begin{array}{l}\text { Fuerza explosiva tren inferior } \\
(\mathrm{cm})\end{array}$ & 141,31 & 21,64 \\
Velocidad/agilidad (segundos) & 14,68 & 1,38 \\
Vo ${ }_{2}$ max $(\mathrm{ml} / \mathrm{kg} / \mathrm{min})$ & 44,06 & 3,91 \\
\hline
\end{tabular}

En el análisis de los resultados estratificados por sexo, en relación con el estado nutricional, se pudo evidenciar que el 73,36\% de las niñas se encontraban dentro de una Zona Saludable. Algo similar ocurrió en el cumplimiento de las recomendaciones sobre actividad física para la salud propuesta por la OMS, encontrando que el $47,62 \%$ de las niñas realizaban sesenta minutos de actividad física, por lo menos durante cinco días a la semana. Con respecto a los resultados encontrados en la evaluación de los niveles de la condición física, los niños obtuvieron mejores resultados en las pruebas de fuerza de prensión manual $(\mathrm{p}=0,045)$, fuerza explosiva de tren inferior $(\mathrm{p}=0,003)$, velocidad/agilidad $(\mathrm{p}<0,001)$ y capacidad aeróbica $(\mathrm{p}=0,001)$ (Tablas 3 y 4$)$.

${ }^{* *} R I$ : Rango Intercuartílico; $¥$ Desviación Estándar.

Tabla 3. Descripción de las características sociodemográficas de actividad física y aptitud física de los participantes según el sexo $(n=115)$

\begin{tabular}{|c|c|c|c|}
\hline Características & $\begin{array}{l}\text { Masculino }(\mathrm{n}=60) \\
\mathrm{n}(\%)\end{array}$ & $\begin{array}{l}\text { Femenino }(\mathrm{n}=55) \\
\mathrm{n}(\%)\end{array}$ & $\mathbf{P}$ \\
\hline Grado & & & 0,753 \\
\hline Tercero primaria & $20(33,33)$ & $17(30,01)$ & \\
\hline Cuarto primaria & $20(33,33)$ & $22(40,00)$ & \\
\hline Quinto primaria & $20(33,33)$ & $16(29,09)$ & \\
\hline Lugar de residencia & & & 0,231 \\
\hline Urbano & $25(59,52)$ & $17(40,48)$ & \\
\hline Rural & $35(47,95)$ & $38(52,05)$ & \\
\hline Estado nutricional (IMC) & & & 0,847 \\
\hline Zona saludable & $43(71,67)$ & $42(76,36)$ & \\
\hline Necesita mejora/riesgo cardiovascular & $13(21,67)$ & $10(18,18)$ & \\
\hline Bajo peso & $4(6,67)$ & $3(5,45)$ & \\
\hline $\begin{array}{l}\text { Cumplimiento de las recomendaciones de la OMS sobre actividad } \\
\text { física para la salud }{ }^{\star}(n=91)\end{array}$ & & & 0,361 \\
\hline Activo & $19(38,78)$ & $20(47,62)$ & \\
\hline Inactivo & $30(61,22)$ & $22(52,38)$ & \\
\hline Realización de actividad física relacionada con el transporte $(\mathbf{n = 9 0 )}$ & & & 0,339 \\
\hline No realiza & $15(31,25)$ & $15(35,71)$ & \\
\hline Entre menos de 10 minutos y 30 minutos & $26(54,17)$ & $19(45,24)$ & \\
\hline Más de 30 minutos & $7(14,58)$ & $8(19,05)$ & \\
\hline Conducta sedentaria $(n=90)$ & & & 0,359 \\
\hline Menos de una hora al día - 4 horas al día & $41(85,42)$ & $36(85,71)$ & \\
\hline Cinco horas al día - 8 horas al día & $4(8,33)$ & $6(14,29)$ & \\
\hline Más de 8 horas al día & $3(6,25)$ & $0(0,00)$ & \\
\hline
\end{tabular}


Tabla 4. Descripción de las características sociodemográficas de actividad física y aptitud física de los participantes según el sexo $(n=115)$

\begin{tabular}{|c|c|c|c|}
\hline Características & $\begin{array}{c}\text { Masculino }(\mathrm{n}=60) \\
\text { Promedio (DE) / Mediana } \dagger(\mathrm{RI})\end{array}$ & $\begin{array}{c}\text { Femenino }(\mathrm{n}=55) \\
\text { Promedio }(\mathrm{DE}) / \text { Mediana } \dagger(\mathrm{RI})\end{array}$ & $\mathbf{P}$ \\
\hline Edad & $9,88(1,23)$ & $9,67(1,08)$ & 0,336 \\
\hline Talla $(\mathrm{cm})$ & $136,96(7,29)$ & $139,18(7,56)$ & 0,113 \\
\hline Peso (kg) & $32,81(6,93)$ & $33,80(8,20) \dagger$ & 0,187 \\
\hline $\operatorname{IMC}\left(\mathrm{kg} / \mathrm{m}^{2}\right)$ & $16,65(2,85) \dagger$ & $17,10(3,50) \dagger$ & 0,620 \\
\hline Fuerza de prensión manual $(\mathrm{kg})$ & $16,00(4,50) \dagger$ & $15,29(3,59)$ & 0,045 \\
\hline Fuerza explosiva tren inferior $(\mathrm{cm})$ & $146,90(23,71)$ & $135,21(17,38)$ & 0,003 \\
\hline Velocidad/agilidad (segundos) & $14,08(1,22)$ & $15,34(1,24)$ & $<0,001$ \\
\hline $\mathrm{Vo}_{2} \max (\mathrm{ml} / \mathrm{kg} / \mathrm{min})$ & $45,42(3,97)$ & $42,57(3,27)$ & 0,001 \\
\hline
\end{tabular}

IMC: Índice de Masa Corporal; ${ }^{\star}$ Determinado por la Encuesta Mundial de Salud a Escolares (EMSE); ${ }^{\star *}$ RI: Rango Intercuartílico; ‡Desviación Estándar.

Al hacer la estratificación de los resultados por el lugar de residencia, se observó que en comparación a los datos encontrados en los niños de la zona rural, existía un porcentaje $(63,46 \%)$ mayor de escolares que vivían en la zona urbana que cumplen las recomendaciones de actividad física para la salud $(\mathrm{p}<0,001)$. Con respecto a la aptitud física, se pudo establecer que los niños que habitan en la zona rural obtuvieron mejores niveles de condición física en las pruebas de fuerza de prensión manual $(\mathrm{p}=0,177)$, fuerza explosiva de tren inferior $(\mathrm{p}=0,277)$ y capacidad aeróbica $(\mathrm{p}=0,402)$, pero no se encontraron diferencias estadísticamente significativas (Tablas 5 y 6 ).

\section{DISCUSIÓN}

A partir de este estudio se pudieron establecer los niveles de aptitud física orientada a la salud en estudiantes escolarizados del Colegio Integrado Nuestra Señora de las Mercedes del municipio de Lebrija. También se pudieron establecer algunos indicadores del cumplimiento de las recomendaciones de actividad física propuestas por la OMS, la cantidad de tiempo que utilizan para desplazarse de su hogar a la institución educativa y la cantidad de tiempo en la que llevan conductas sedentarias. Este estudio permitió identificar las características específicas en relación con los factores mencionados anteriormente, clasificando la población evaluada según el sexo y zona de residencia.
En relación con la inactividad física, el 57,14\% de los estudiantes participantes reportaron la realización de menos de 300 minutos de actividad física a la semana. Los resultados registrados en este estudio son inferiores a los resultados obtenidos en escolares de cinco ciudades de Colombia, donde se registraron prevalencias de inactividad física superiores al $75 \%$ utilizando el cuestionario EMSE ${ }^{13}$. En un estudio de las mismas características, realizado en Argentina ${ }^{18}$, se registraron los datos obtenidos a partir de la Encuesta Mundial de Salud Escolar, obteniendo como resultado que el $83,30 \%$ de la población evaluada se caracteriza como físicamente inactivos. En relación con el género, los niños del presente estudio presentan mayores niveles de inactividad física que las niñas $(61,22 \%$ contra 52,38\%) siendo esta cifra contraria a los datos obtenidos por Piñeros y Pardo, en los que las mujeres fueron físicamente más inactivas en cada uno de los grupos donde se realizó el proceso de medición ${ }^{12}$.

Respecto a la actividad física, según el lugar de residencia, se pudo establecer que los escolares que habitan en el sector urbano son físicamente más activos que los que viven en el sector rural. Estos resultados concuerdan con los reportados por Joens-Matre y colaboradores, quienes compararon los niveles de actividad física de niños que viven en zonas urbanas, niños que viven en ciudades pequeñas y niños que viven en zonas rurales, encontrando que los niños que viven en ciudades pequeñas son físicamente más activos ${ }^{8}$. 
Tabla 5. Descripción de las características sociodemográficas de actividad física y condición física de los participantes según el lugar de residencia $(\mathrm{n}=115)$

\begin{tabular}{|c|c|c|c|}
\hline Características & $\begin{array}{c}\text { Urbano }(n=73) \\
\text { n (\%) }\end{array}$ & $\begin{array}{c}\text { Rural (n=42) } \\
\text { n (\%) }\end{array}$ & $\mathbf{P}$ \\
\hline Sexo & & & 0,231 \\
\hline Masculino & $38(52,05)$ & $17(40,48)$ & \\
\hline Femenino & $35(47,95)$ & $25(59,52)$ & \\
\hline Grado & & & 0,801 \\
\hline Tercero primaria & $22(30,14)$ & $15(35,71)$ & \\
\hline Cuarto primaria & $28(38,36)$ & $14(33,33)$ & \\
\hline Quinto primaria & $23(31,51)$ & $13(30,95)$ & \\
\hline Estado nutricional (IMC) & & & 0,434 \\
\hline Zona saludable & $54(73,97)$ & $31(73,81)$ & \\
\hline Necesita mejora/riesgo cardiovascular & $16(21,92)$ & $7(16,67)$ & \\
\hline Bajo peso & $3(4,11)$ & $4(9,52)$ & \\
\hline $\begin{array}{l}\text { Cumplimiento de las recomendaciones de la OMS sobre actividad } \\
\text { física para la salud }{ }^{*}(n=91)\end{array}$ & & & $<0,001$ \\
\hline Activo & $33(63,46)$ & $6(15,38)$ & \\
\hline Inactivo & $19(36,54)$ & $33(84,62)$ & \\
\hline Realización de actividad física relacionada con el transporte $(n=90)$ & & & $<0,001$ \\
\hline No realiza & $10(19.61)$ & $20(51,28)$ & \\
\hline Entre menos de 10 minutos y 30 minutos & $33(64.71)$ & $12(30,77)$ & \\
\hline Más de 30 minutos & $8(15.69)$ & $7(17,95)$ & \\
\hline Conducta sedentaria $(\mathrm{n}=90)$ & & & 0,009 \\
\hline Menos de 1 hora al día - 4 horas al día & $39(76,47)$ & $38(97,44)$ & \\
\hline Cinco horas al día - 8 horas al día & $9(17,65)$ & $1(2,56)$ & \\
\hline Más de 8 horas al día & $3(5,88)$ & $0(0,00)$ & \\
\hline
\end{tabular}

Tabla 6. Descripción de las características sociodemográficas de actividad física y condición física de los participantes según el lugar de residencia $(\mathrm{n}=115)$

\begin{tabular}{|c|c|c|c|}
\hline Características & $\begin{array}{c}\text { Urbano }(\mathrm{n}=73) \\
\text { Promedio }(\mathrm{DE}) / \text { Mediana } \dagger \\
(\mathrm{RI})\end{array}$ & $\begin{array}{c}\text { Rural }(\mathrm{n}=42) \\
\text { Promedio (DE) / Mediana } \dagger \\
(\mathrm{RI})\end{array}$ & $\mathbf{P}$ \\
\hline Edad & $9,79(1,10)$ & $9,76(1,28)$ & 0,886 \\
\hline Talla $(\mathrm{cm})$ & $138,20(7,40)$ & $137,71(7,68)$ & 0,736 \\
\hline Peso (kg) & $33,00(8,20) \dagger$ & $32,55(6,54)$ & 0,303 \\
\hline $\mathrm{IMC}\left(\mathrm{kg} / \mathrm{m}^{2}\right)$ & $17,10(2,90) \dagger$ & $17,04(2,58)$ & 0,120 \\
\hline Fuerza de prensión manual (kg) & $15,73(3,55)$ & $16,95(4,97)$ & 0,177 \\
\hline Fuerza explosiva tren inferior $(\mathrm{cm})$ & $139,64(22,29)$ & $144,21(20,40)$ & 0,277 \\
\hline Velocidad/agilidad (segundos) & $14,67(1,42)$ & $14,70(1,33)$ & 0,925 \\
\hline $\mathrm{Vo}_{2} \max (\mathrm{ml} / \mathrm{kg} / \mathrm{min})$ & $43,82(4,07)$ & $44,46(3,62)$ & 0,402 \\
\hline
\end{tabular}

IMC: Índice de Masa Corporal; ${ }^{\star}$ Determinado por la Encuesta Mundial de Salud a Escolares (EMSE); ${ }^{\star *}$ RI: Rango Intercuartílico; $¥$ Desviación Estándar. 
Como lo indica Gutiérrez y colaboradores una posible explicación a este hecho es que la impresión que se tiene del entorno donde se vive está relacionada directamente con los hábitos de actividad física de los escolares. Por esta razón, hay que tener en cuenta que factores como el tipo de urbanización en el que se vive, la cercanía, la percepción de seguridad y facilidad de acceso que tenga a la institución educativa y a los lugares adecuados para realizar actividad física, pueden facilitar u obstaculizar la realización de actividad física ${ }^{19}$.

Lebrija se caracteriza por ser un municipio con una amplia extensión rural y con un área urbana reducida, donde hay una similitud considerable entre la cantidad de personas habitantes de cada zona, con una población urbana de 18.747 habitantes y una población rural de 19.813 habitantes ${ }^{20}$. Por lo tanto, se puede concluir que debido a la amplia extensión de tierra de la zona rural en comparación a la extensión de la zona urbana, se puede hacer un análisis con respecto a la cercanía de instalaciones deportivas, instituciones educativas y los hogares de los vecinos del mismo lugar de residencia, entendiendo que son diferentes las distancias que hay que recorrer para llegar a un determinado lugar a realizar actividad física, siendo este un argumento que confirma lo descrito por Gutiérrez-Zornoza y colaboradores en su estudio, donde se demuestra que debido a la cercanía de los lugares adecuados para la práctica de actividad física, es más fácil para los niños de la zona urbana acceder a realizar actividad física ${ }^{19}$.

Respecto a la actividad física relacionada con el transporte, se pudo determinar que el $50 \%$ de las personas encuestadas caminan entre menos de diez minutos y treinta minutos para desplazarse de su hogar hasta el colegio y viceversa. No se encontraron diferencias estadísticamente significativas por género $(\mathrm{p}=0,339)$. Sin embargo, se pudo evidenciar una gran diferencia entre las prevalencias en la realización de actividad física como medio de transporte entre los habitantes de zonas urbanas con $80,40 \%$ y los de zonas rurales con $48,72 \%(\mathrm{p}<0,001)$. Estos resultados confirman lo argumentado por Gutiérrez-Zornoza y colaboradores donde se afirma que la cantidad de niños que se desplazan al colegio con actividad física depende de la ubicación de las instituciones educativas y de la seguridad que tenga el camino para llegar a este lugar ${ }^{19}$. Al hacer el análisis de las distancias que hay que recorrer para llegar a los centros educativos en las zonas urbanas y en las zonas rurales, se puede entender que es mucho más fácil para los niños de la zona urbana desplazarse caminando hacia el colegio, además que no implica un gran riesgo hacerlo por este medio. Esta situación no ocurre con los niños de la zona rural, que tienen que recorrer distancias más largas, y sin la compañía de un adulto su seguridad puede estar en riesgo.

En cuanto al tiempo de conducta sedentaria, se encontró que el $85,55 \%$ de las personas encuestadas gastan entre menos de una hora y cuatro horas diarias haciendo algún tipo de actividad que no implica ningún gasto energético. Al hacer la comparación por género ( $\mathrm{p}=0,359)$ no se encontraron diferencias estadísticamente significativas. En la comparación por lugar de residencia se encontró que los niños que habitan en zonas urbanas realizan durante más tiempo actividades sedentarias, encontrando que el $23,53 \%$ de los niños de la zona urbana invierten entre cinco o más horas diarias en la realización de actividades que no impliquen gasto energético $(\mathrm{p}=0,009)$.

En referencia al estado nutricional, el 73,95\% de la población evaluada se encuentra dentro de una zona saludable, según criterios de Fitnessgram ${ }^{\oplus 21}$, siendo este porcentaje mayor en las niñas $(76,36 \%)$ que en los niños $(71,67 \%)$, sin encontrar diferencias significativas $(\mathrm{p}=0,847)$. Estos resultados son similares a los reportados por Davis y colaboradores en Estados Unidos $^{22}$, y Ferrante y colaboradores en Argentina ${ }^{18}$. Una posible explicación a este hecho es la relación que existe entre el cumplimiento de las recomendaciones de actividad física por parte de la población estudiada, debido a que, en un estudio realizado por Villagrán Pérez y colaboradores en España, se obtuvieron datos que contrastan con los datos de este estudio, donde los niños presentaron un mayor porcentaje de personas dentro de la zona saludable que las niñas. Mientras tanto, en el mismo estudio se encontró que los escolares varones tenían una diferencia estadística amplia con respecto al cumplimiento de las recomendaciones de actividad sobre las mujeres $(71,7 \% \text { contra } 46,0 \%)^{23}$.

$\mathrm{Al}$ analizar el estado nutricional según el lugar de residencia, se registró que el 21,92\% de escolares de la zona urbana y el $16.67 \%$ de las zonas rurales necesitan 
una mejora (sobrepeso) o tienen riesgo cardiovascular (obesidad), sin embargo, no se encontraron diferencias estadísticamente significativas entre ambos grupos $(\mathrm{p}=0,434)$. Estos porcentajes son similares a los encontrados por Davis y colaboradores ${ }^{22}$.

En la prueba de fuerza prensión manual los niños tuvieron una media de $16 \mathrm{~kg}$ y las niñas de $15,29 \mathrm{~kg}$ $(\mathrm{p}=0,045)$, con diferencias estadísticamente significativas. Se encontraron resultados similares en un estudio realizado por Chillón en España ${ }^{24}$. En otro estudio desarrollado en este mismo país por Cuenca-García y colaboradores ${ }^{5}$ se encontró que la media de los datos obtenidos en niños y niñas fue menor, mientras que los datos obtenidos por Rodríguez y colaboradores en Colombia ${ }^{25}$, dan como resultado una media con valores más altos (tanto en hombres como en mujeres) al resultante del presente estudio.

En la prueba de fuerza explosiva de tren inferior o de salto de longitud, los niños obtuvieron una media de $146,9 \mathrm{~cm}$ y las niñas de $135,21 \mathrm{~cm}$, encontrado diferencias estadísticamente significativas entre ambos $(\mathrm{p}=0,003)$. Los datos obtenidos se pueden comparar con otros estudios, al encontrar que los valores alcanzados por los escolares del municipio de Lebrija son superiores tanto en niños como en niñas, a los recogidos en las investigaciones de Chillón y colaboradores $^{24}$, Cuenca-García y colaboradores ${ }^{5}$, Rodríguez Valero y colaboradores ${ }^{25}$ y Secchi y colaboradores ${ }^{26}$.

En la prueba realizada de velocidad/agilidad $4 \times 10$ $\mathrm{m}$, entre los datos obtenidos por los niños $(14,08 \mathrm{se}-$ gundos) y las niñas (15,34 segundos) se obtuvo una diferencia estadísticamente significativa entre cada grupo poblacional $(\mathrm{p}<0,001)$. Al comparar los datos estudiados por Secchi y cols. en Argentina ${ }^{26}$, se puede concluir que, al hacer el análisis de los resultados por género, los datos obtenidos son similares a los de este estudio obteniendo los niños un puntaje superior al de las niñas.

Los resultados obtenidos de la prueba de capacidad aeróbica se relacionan por medio del consumo de oxígeno, el cual varía dependiendo de la edad, el género y los niveles alcanzados en la prueba de ida y vuelta de 20 metros. Los niños lograron un resultado superior con un $\mathrm{VO}_{2}$ máx. de $45,42 \mathrm{ml} / \mathrm{kg} / \mathrm{min}$, mientras que en las niñas fue de $42,57 \mathrm{ml} / \mathrm{kg} / \mathrm{min}$. Estos re- sultados van en relación con los datos encontrados en investigaciones realizadas por Secchi y colaboradores, Cuenca-García y colaboradores, y Palomino-Devia y colaboradores, donde en todos los casos los niños obtuvieron un mayor resultado en el consumo máximo de oxígeno ${ }^{5,26,27}$.

En relación con la aptitud física, los habitantes de zonas rurales obtuvieron mejores resultados en las pruebas de fuerza de prensión manual $(16,95 \mathrm{~kg}$ contra $15,73 \mathrm{~kg}, \mathrm{p}=0,177)$, fuerza explosiva de tren inferior $(144,21$ contra $139.64, \mathrm{p}=0,277)$ y en la capacidad aeróbica $(44,46 \mathrm{ml} / \mathrm{kg} / \mathrm{min}$ contra $43,82 \mathrm{ml} / \mathrm{kg} / \mathrm{min}$, $\mathrm{p}=0,402)$. Por otra parte, los participantes evaluados de la zona urbana tuvieron mejores resultados en la prueba de velocidad/agilidad (14,67 segundos contra 14,70 segundos, $\mathrm{p}=0,925)$. Estos resultados van en línea con los hallados por De la Cruz y colaboradores, en España ${ }^{28}$.

En la evaluación de la aptitud física por medio de la batería ALPHA-Fitness se obtuvieron resultados que pueden ser comparados con otros estudios realizados en igualdad de condiciones. Al hacer el análisis de los resultados por género en la evaluación de la condición física, se encontró que los hombres lograron resultados superiores en la totalidad de las pruebas.

En relación con los datos obtenidos al hacer la comparación por sexo, Hoyo Lora y Sañudo plantean que las diferencias entre la aptitud física de los niños y niñas pueden estar relacionados con factores socioculturales, donde los niños participan más de actividades deportivas y recreativas, mientras que las niñas se les introduce a actividades más de tipo sedentario ${ }^{29}$. $\mathrm{Al}$ respecto de esto, Zaragoza Casterad y colaboradores exponen que, más allá del tiempo que utilicen en actividad física, los hombres tienden a realizar actividades de mayor intensidad que las mujeres, presentando normalmente un mejor rendimiento y una mayor motivación a la hora de realizar pruebas de condición física ${ }^{7}$. De la misma forma, Moreno y colaboradores sugieren que la mayor cantidad de las mujeres tienden a auto-percibirse menos competentes y con menores capacidades en relación con los hombres, por sentir menos diversión y disfrute hacia la actividad física en general ${ }^{30}$.

A pesar de que los resultados obtenidos fueron favorables para los escolares de zonas rurales, las diferencias no fueron estadísticamente significativas. 
Como lo dicen De la Cruz y colaboradores es complicado establecer unos parámetros claros con base en los resultados obtenidos en la comparación de la aptitud física entre entornos rurales y urbanos, si no se establecen criterios claros en relación con el tamaño de la población en el área rural y urbana ${ }^{28}$. Una posible explicación a la baja diferencia estadística entre los resultados encontrados es la cercanía y la similitud que existe entre una población y otra, al ser Lebrija un municipio que es en su mayor parte rural.

Para concluir, el presente estudio permitió establecer los niveles de aptitud física de los escolares de zonas urbanas y rurales del Colegio Integrado Nuestra Señora de las Mercedes. A pesar de que existen numerosas investigaciones que demuestran que el entorno donde se vive puede afectar diferentes factores en relación con la vida de las personas, al hacer la estratificación por lugar de residencia, no se encontraron diferencias estadísticamente significativas en las variables relacionadas con la aptitud física. Esto puede ocurrir debido a la proximidad que hay entre las zonas de residencia evaluadas, pudiendo existir grandes similitudes en los estilos de vida de ambas poblaciones.

Una de las debilidades del presente estudio fue el tipo de muestreo, debido a que se realizó un muestreo por conveniencia. Además de esto, se recomienda aumentar el tamaño de la muestra en futuras investigaciones, con el fin de obtener resultados más precisos y seguros a la hora de hacer el análisis estadístico.

Se puede destacar como fortaleza del estudio, el uso de instrumentos de medición validados, como lo son la Encuesta Mundial de Salud Escolar (EMSE) y la batería ALPHA Fitness, permitiendo comparar los resultados del presente estudio con investigaciones realizadas en diferentes países.

\section{REFERENCIAS}

1. Vallejo AP, González JM. Estudio sobre hábitos de actividad física saludable en niños de educación primaria de Jaén capital. Apuntes Educación Física y Deportes. 2012;107:13-23.

2. Ramírez W, Vinaccia S, Gustavo RS. El impacto de la actividad física y el deporte sobre la salud, la cognición, la socialización y el rendimiento académico: una revisión teórica. Revista de Estudios Sociales. 2004;18:67-75.
3. Loucaides CA, Chedzoy SM, Bennett N. Differences in physical activity levels between urban and rural school children in Cyprus. Health Educ Res. 2004;19(2):138147. DOI: 10.1093/her/cyg014.

4. Caspersen CJ, Powell KE, Christenson GM. Physical activity, exercise, and physical fitness: definitions and distinctions for health-related research. Public Health Rep. 1985;100(2):126-131.

5. Cuenca-García M, Jiménez-Pavón D, España-Romero V, Artero E, Castro-Piñero J, Ortega F, et al. Condición física relacionada con la salud y hábitos de alimentación en niños y adolescentes: propuesta de addendum al informe de salud escolar. Revista de Investigación en Educación. 2011;9(2):35-50.

6. Garzón MJC, Ortega Porcel F, Ruiz JR. Mejora de la forma física como terapia antienvejecimiento. Medicina Clínica. 2005;124(4):146-155.

7. Zaragoza Casterad J, Serrano Ostariz E, Generelo Lanaspa E. Dimensiones de la condición física saludable: evolución según edad y género. Revista Internacional de Medicina y Ciencias de la Actiivdad Física y del Deporte. 2005;5(17):50-67.

8. Joens-Matre RR, Welk GJ, Calabro MA, Russell DW, Nicklay E, Hensley LD. Rural-urban differences in physical activity, physical fitness, and overweight prevalence of children. J Rural Health. 2008;24(1):49-54. DOI: 10.1111/j.1748-0361.2008.00136.x.

9. Karkera A, Swaminathan N, Pais SM, Vishal K, Rai S. Physical fitness and activity levels among urban school children and their rural counterparts. Indian J Pediatr. 2014;81(4):356-361. DOI: 10.1007/s12098-013-1033-8

10. Peña Reyes ME, Tan SK, Malina RM. Urban-rural contrasts in the physical fitness of school children in Oaxaca, Mexico. Am J Hum Biol. 2003;15(6):800-813. DOI: 10.1002/ajhb.10218.

11. Torres-Luque G, Carpio E, Sánchez AL, Sánchez MLZ. Niveles de condición física de escolares de educación primaria en relación a su nivel de actividad física y al género. Retos. Nuevas Tendencias en Educación Física, Deporte y Recreación. 2014;(25):17-22.

12. Piñeros M, Pardo C. Actividad física en adolescentes de cinco ciudades colombianas: resultados de la Encuesta Mundial de Salud a Escolares. Rev Salud Pública. 2010;12(6):903-914.

13. Organización Mundial de la Salud. La Encuesta Mundial de Salud a Escolares (GSHS) Objeto y Metodología. [Internet] 2018. [Fecha de acceso: febrero 23 de 2018]. URL 
disponible en: http://www.who.int/ncds/surveillance/ gshs/methodology/es/

14. Ruíz JR, España Romero V, Castro Piñero J, Artero EG, Ortega F, Cuenca-García M, Castillo MJ. Batería ALPHA-Fitness: test de campo para la evaluación de la condición física relacionada con la salud en niños y adolescentes. Nutr Hosp. 2011;26(6):1210-1214. DOI: 10.3305/nh.2011.26.6.5611.

15. Ruiz JR, España Romero V, Ortega FB, Sjostrom M, Castillo MJ, Gutiérrez A. Hand span influences optimal grip span in male and female teenagers. J Hand Surg Am. 2006:31(8):1367-1372. DOI: 10.1016/j.jhsa.2006.06.014

16. Ruiz JR, Romero VE, Piñero JC, Artero EG, Ortega FB. Manual de Instrucciones Batería ALPHA-Fitness. Nutr Hosp. 2011;26 (6):1210-1214.

17. Leger LA, Mercier D, Gadoury C, Lambert J. The multistage 20-meter shuttle run test for aerobic fitness. J Sports Sci. 1988;6(2):93-101. DOI: 10.1080/02640418808729800.

18. Ferrante D, Linetzky B, Ponce M, Goldberg L, et al. Prevalencia de sobrepeso, obesidad, actividad física y tabaquismo en adolescentes argentinos: Encuestas Mundiales de Salud Escolar y de Tabaco en Jóvenes, 2007-2012. Arch Arg Pediatr 2014;112(6):496-503.

19. Gutiérrez-Zornoza, M., Rodríguez-Martín, B., Martínez-Andrés, M., García-López, U., Sánchez-López, M. Percepción del entorno para la práctica de actividad física en escolares de la provincia de Cuenca, España. Gaceta Sanitaria 2014;28(1):34-40. DOI: 10.1016/j.gaceta.2013.04.011.

20. Municipio de Lebrija Santander. Mi Municipio. Información del municipio. 2016. URL disponible en: http://www. lebrija-santander.gov.co/Paginas/default.aspx

21. Plowman SA, Meredith MD. Fitnessgram/Activitygram Reference Guide ( $4^{\text {th }}$ Edition). Dallas, TX: The Cooper Institute; 2013.

22. Davis AM, Bennett KJ, Befort C, Nollen N. Obesity and related health behaviors among urban and rural children in the United States: data from the National Health and Nutrition Examination Survey 2003-2004 and 2005-2006. J Pediatr Psychol. 2011;36(6):669-676. DOI: $10.1093 /$ jpepsy/jsq117.
23. Villagrán Pérez S, Rodríguez-Martín A, Novalbos Ruiz JP, Martínez Nieto JM, Lechuga Campoy JL. Hábitos y estilos de vida modificables en niños con sobrepeso y obesidad. Nutr Hosp. 2010;25(5):823-831.

24. Chillón P, Ortega FB, Ferrando JA, Casajus JA. Physical fitness in rural and urban children and adolescents from Spain. J Sci Med Sport. 2011;14(5):417-423. DOI: 10.1016/j.jsams.2011.04.004.

25. Rodríguez Valero FJ, Gualteros JA, Torres JA, Umbarila Espinosa LM, Ramírez Vélez R. Asociación entre el desempeño muscular y el bienestar físico en niños y adolescentes de Bogotá, Colombia. Nutr Hosp. 2015;32(4):15591566. DOI: $10.3305 /$ nh.2015.32.4.9310

26. Secchi JD, García GC, España-Romero V, Castro-Piñero J. Condición física y riesgo cardiovascular futuro en niños y adolescentes argentinos: una introducción de la batería ALPHA. Arch Arg Pediatr. 2014;112(2):132-144.

27. Palomino-Devia C, González-Jurado JA, Ramos-Parraci CA. Composición corporal y condición física de escolares colombianos de educación secundaria y media de Ibagué. Biomédica. 2017;37(3):408-415. DOI: 10.7705/biomedica.v37i3.3455.

28. De la Cruz Sánchez E, Aguirre-Gómez MD, Pino-Ortega J, Díaz-Suárez A, Valero Valenzuela A, García-Pallarés J. Diferencias en la condición física en niños de entornos rurales y urbanos. Revista de Psicología del Deporte. 2012;21(2):359-363.

29. Hoyo Lora MD, Sañudo Corrales FB. Motivos y hábitos de práctica de actividad física en escolares de 12 a 16 años en una población rural de Sevilla. Revista Internacional de Medicina y Ciencias de la Actividad Física y del Deporte. 2007:7(26): 87-98.

30. Moreno Murcia JA, Martínez Galindo C, Alonso Villodre N. Actitudes hacia la práctica físico-deportiva según el sexo del practicante. Revista Internacional de Ciencias del Deporte. 2006:3(2):20-43.

\section{Correos electrónicos de los autores:}

Diego Fernando Álvarez Villafrade: diegoalvillaf@gmail.com Luis Gabriel Rangel Caballero: dcultu@ustabuca.edu.co 